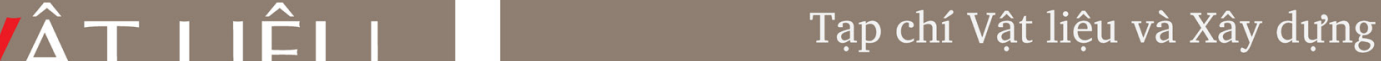 \\ ISSN $1859-381 \mathrm{X}$ \\ Website: www. jomc.vn
}

\section{Nghiên cứu tính năng nhiệt của hệ thống cửa sổ, cửa đi và kết cấu che nắng}

Nguyễn Thị Thùy Linh' ${ }^{1}$ Lê Thị Song ${ }^{1}$, Lê Cao Chiến ${ }^{1}$

${ }^{1}$ Trung tâm Thiết bị, Môi trường và An toàn lao động, Viện Vật liệu xây dựng, ngõ 235 Nguyễn Trãi, p. Thanh Xuân Trung, q. Thanh Xuân, Hà Nội

TỪ KHOÁ

Cửa sổ

Cửa đi

Kết cấu che nắng

U-value

SHGC

VLT

WINDOW

THERM

\section{TÓM TẮT}

Hệ thống cửa sổ, cửa đi và kết cấu che nắng thường được biết tới là một phần quan trọng trong kết cấu toà nhà, đảm bảo che chắn nắng, gió, mưa bão và bảo vệ con người khi ở bên trong. Ngoài những vai trò đó thì hệ thống cửa sổ, cửa đi còn tác động tới việc tiêu thụ năng lượng của tòa nhà. Các tác động đó có thể xác định thông qua thông số nhiệt và quang học của toàn bộ kết cấu cửa sổ, cửa đi, bao gồm: độ truyền nhiệt U-value; độ hấp thụ năng lượng mặt trời (SHGC) và độ truyền sáng (VLT). Các thông số truyền nhiệt này qua mỗi sản phẩm cửa là một hàm thuật toán rất phức tạp của sự chênh lệch nhiệt độ giữa bên trong và bên ngoài và cường độ bức xạ năng lượng mặt trời trên sản phẩm. Để đơn giản hóa và tối ưu hóa quá trình tính toán, tránh việc tính toán bằng thủ công có thể gây ra sai số do nhầm lẫn, khó kiểm soát và khó tiếp cận tính toán, Phòng thí nghiệm Lawrence Berkeley National Laboratory (Hoa kỳ) đã phát triển các phần mềm tính toán là OPTICS, WINDOW và THERM. Giá trị tính toán bằng phần mềm với giá trị công bố trong tiêu chuẩn đảm bảo sai lệch không quá $3 \%$ đáp ứng các tiêu chuẩn tính toán TCVN 11857, ISO 15099, ISO 10077; ANSI/NFRC 200-2020.

\section{ABSTRACT}

A window, door and shading device system is known for its significant part of the building structure, ensuring protection from the sun, wind, rain and storms and protecting people living inside. In addition, the window, door and shading device system also affects the energy consumption of the building. These effects can be determined through thermal and optical properties of the whole system, include: thermal transmittance (U-value), solar heat gain coefficient (SHGC) and visible transmittance (VLT). The thermal and optic properties through each door product are extremly complex algorithmic formula of the temperature difference between the inside and outside the building and the solar radiation intensity on the product. Lawrence Berkeley National Laboratary (USA) has developed the calculation software as: OPTICS, WINDOW and THERM, to simplify and optimize the calculation process, avoiding errors of confusion, difficult to control and approach the formula from manual calculation. The results show that the value calculated by the two solfware compared with the value announced in the standards TCVN 11857, ISO 15099, ISO 10077; ANSI/NFRC 200-2020 is not more than $3 \%$ to meet the calculation standards.

\section{Giới thiệu}

Tại Việt Nam, tổng mức tiêu thụ năng lượng của các công trình dân dụng chiếm gần 40 \% tổng mức tiêu thụ năng lượng của quốc gia, trong khi đó mỗi năm có khoảng 80 đến 90 triệu $\mathrm{m}^{2}$ sàn công trình được xây mới. Nhu cầu tiêu thụ năng lượng ngày càng nhiều đồng nghĩa với lượng phát thải khí nhà kính ngày càng gia tăng. Trước thách thức này, những giải pháp tiết kiệm năng lượng cho tòa nhà, giải pháp xây dựng môi trường sống xanh ngày càng được chú trọng và phát triển với mục tiêu giảm lượng phát thải khí nhà kính, bảo vệ môi trường, đáp ứng nhu cầu thực tiễn của con người. Nhiều hoạt động đã được triển khai, tập trung vào thiết kế tòa nhà với các tính năng tận dụng ánh sáng, gió tự nhiên và vật liệu có tính cách nhiệt tốt, ví dụ tòa nhà Trung tâm Công nghệ cao bưu chính viễn thông ứng dụng hệ thống rèm che đi kèm với cửa sổ sử dụng kính hộp Low-E có hệ số bức xạ thấp, phản xạ cao giúp điều chỉnh ánh sáng tự nhiêm, giảm lượng nhiệt hấp thụ vào mùa hè và hạn chế thất thoát nhiệt vào mùa đông [11].

Ở các thành phố đông đúc tại Hàn Quốc, trung bình 90,9 \% lượng phát thải khí nhà kính đến từ việc tiêu thụ năng lượng, trong đó $68,5 \%$ là từ việc sử dụng điều hòa và sưởi ấm của tòa nhà. Nguyên nhân đẩy nhu cầu sử dụng điều hòa và sưởi ấm tăng cao là do sự truyền nhiệt từ bên ngoài vào bên trong nhà thông qua tường, đặc biệt là qua cửa sổ do có thông số đặc trưng nhiệt thấp và độ truyền nhiệt (U-value) cao. Nghiên cứu chỉ ra rằng tính chất vật liệu và thiết kế của cửa sổ có tác động tới sự tăng giảm của độ truyền nhiệt (U-value), cho thấy kết quả đo U-value với các loại cửa sổ khác nhau. Loại cửa lắp lớp kính Low-E ra phía ngoài có thông số U-value đo được là $1,794 \mathrm{~W} / \mathrm{m}^{2} \mathrm{~K}$ và $0,9165 \mathrm{~W} / \mathrm{m}^{2} \mathrm{~K}$ thấp hơn nhiều so với loại cửa lắp lớp kính LowE vào phía trong nhà với U-value là $2,688 \mathrm{~W} / \mathrm{m}^{2} \mathrm{~K}$, do kính Low-E có tính năng 
phản xạ lại ánh nắng mặt trời, phát xạ nhiệt chậm, làm giảm sự phát tán và hấp thụ nhiệt lượng. Cửa có càng nhiều lớp kính thì độ truyền nhiệt càng thấp [2].

Tại Hong Kong, nhiệt độ ngoài trời vào mùa hè cao nhất là $35^{\circ} \mathrm{C}$ và nhiệt độ trong nhà bằng hoặc cao hơn $22^{\circ} \mathrm{C}$, một số nơi có lượng bức xạ mặt trời có thể lớn hơn $1000 \mathrm{~W} / \mathrm{m}^{2}$ nên chỉ cần cửa có độ hấp thụ năng lượng mặt trời (SHGC) cao hơn sẽ làm tăng đáng kể lượng nhiệt hấp thụ của cửa và tăng nhu cầu sử dụng điện làm mát. Nghiên cứu của Siu Fung Fung và Lin Lu cho thấy kết cấu và vật liệu của cửa đóng vai trò quan trọng trong giảm mức hấp thụ nhiệt và sử dụng điện. Với loại cửa xanh xám hai cánh (double sage green) có khả năng làm giảm khoảng 69 \% độ hấp thụ năng lượng mặt trời (SHGC) và giảm $16 \%$ nhu cầu sử dụng điện, tương tự là loại cửa Low-E hai cánh với khoang khí và khoang chân không giúp giảm SHGC lần lượt là $50 \%$ và $10 \%$, độ truyền sáng VLT lên tới 60 \% [3].

Như vậy có thể hiểu các thông số đặc trưng nhiệt của cửa sổ, cửa đi và kết cấu che nắng bao gồm độ truyền nhiệt U-value; độ hấp thụ năng lượng mặt trời (SHGC) và độ truyền sáng (VLT) phản ánh dòng nhiệt truyền qua và phản xạ.

Độ truyền nhiệt (U-value) đại diện cho độ truyền nhiệt của toàn bộ cửa kính, giá trị U-value càng thấp thì khả năng cách nhiệt của cửa càng tốt. Hiểu một cách đơn giản, tổng nhiệt mất đi khi truyền qua hệ thống cửa được tính bằng tích của giá trị U-value với nhiệt độ chênh lệch giữa bên ngoài và bên trong nhà, và với diện tích của toàn bộ cửa. Khi lựa chọn cửa có giá trị U-value thấp bằng một nửa thì tổng nhiệt truyền qua cửa cũng giảm đi một nửa so với ban đầu.

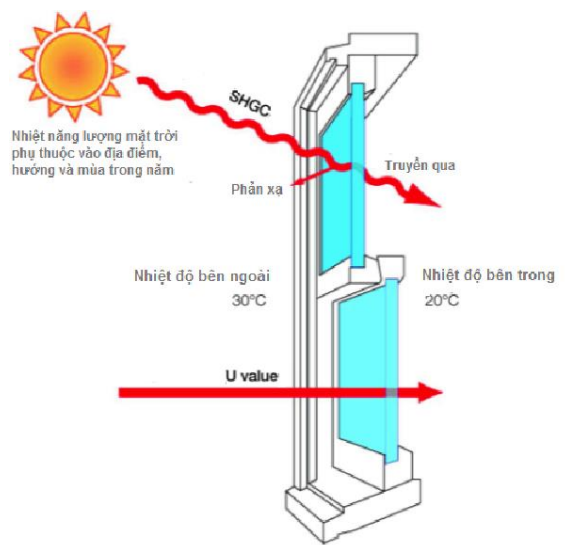

Hình 1. Thông số đặc trưng của lớp cửa kính.

Độ hấp thụ năng lượng mặt trời (SHGC) là nhiệt từ ánh nắng mặt trời chiếu qua hệ thống cửa. Cửa sổ có độ hấp thụ năng lượng mặt trời càng nhỏ thì nhiệt truyền qua hệ thống cửa vào bên trong nhà càng ít. Thông số SHGC chịu ảnh hưởng bởi hướng kính và vị trí của mặt trời, khí hậu và thời gian trong ngày. Do vậy vào ngày nắng, khi ánh nắng mặt trời chiếu vuông góc với cửa kính là lúc đạt độ hấp thụ năng lượng mặt trời mạnh nhất. Khi góc chiếu của ánh nắng mặt trời lớn hơn sẽ làm tăng sự phản xạ và giảm lượng nhiệt truyền qua nhờ diện tích tiếp xúc của cửa với ánh nắng mặt trời bị thu hẹp [8].

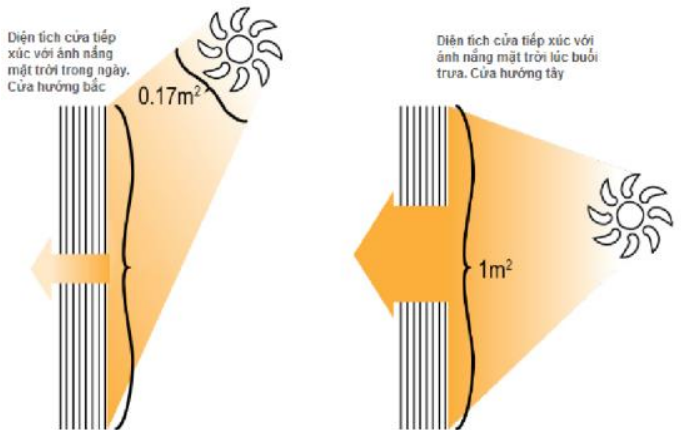

Hình 2. Diện tích tiếp xúc cửa cửa với ánh nắng mặt trời.

Độ truyền sáng (VLT) chính là lượng ánh sáng nhìn thấy được truyền qua cửa. Khi độ truyền sáng thấp sẽ giúp làm giảm sự hấp thụ bức xạ mặt trời nhưng nếu thông số này quá thấp sẽ khiến căn phòng trở nên tối và hệ quả dẫn tới là cần sử dụng đèn điện nhiều hơn và thường xuyên hơn.

Để tính toán độ truyền nhiệt qua sản phẩm cụ thể, hình dạng và kích thước vật lý của sản phẩm phải được thể hiện thông qua bản vẽ thiết kế chi tiết. Điều này bao gồm các đặc tính của kính (đặc tính truyền sáng, đặc tính bức xạ mặt trời và hồng ngoại, và độ dẫn nhiệt), khe hở khí (không khí hoặc khí có độ dẫn điện thấp) các đặc tính nhiệt vật lý, các đặc tính của thanh chia, khung, và điều kiện môi trường. Các thuật toán tiêu chuẩn trong ISO 15099 (TCVN 11857), ISO 10077 để thực hiện tính toán thông số đặc trưng nhiệt của cửa rất phức tạp, dễ gây nhầm lẫn khi tính toán thủ công, do đó nhóm nghiên cứu đã sử dụng các phần mềm của phòng thí nghiệm Lawrence Berkeley National Laboratory (LBNL) bao gồm OPTICS, WINDOW và THERM để đơn giản hóa và tự động hóa quá trình tính toán các thông số đặc trưng nhiệt của cửa.

\section{Vật liệu thử nghiệm, phần mềm tính toán và phương pháp nghiên cứu}

\subsection{Vật liệu thử nghiệm và dữ liệu tính toán}

Mẫu thử là cửa sổ, cửa đi có kết cấu cầu nhiệt dùng loại kính hộp lowE và các bản vẽ chi tiết của sản phẩm (Hình 3).

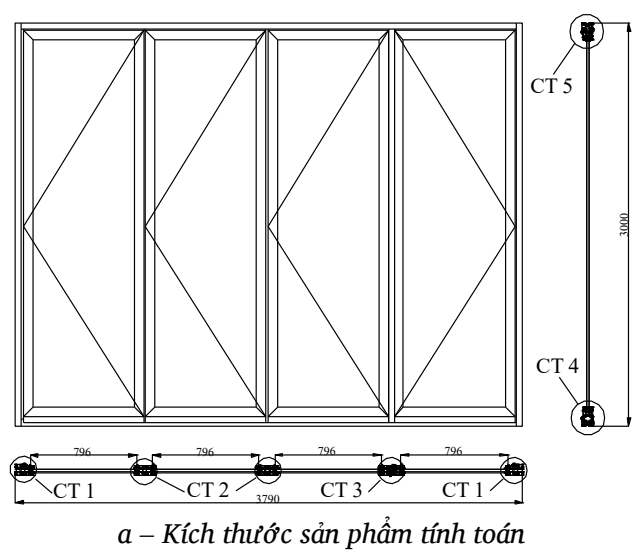




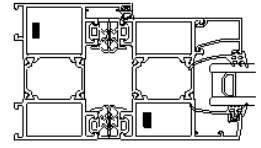

CHI TIET 1

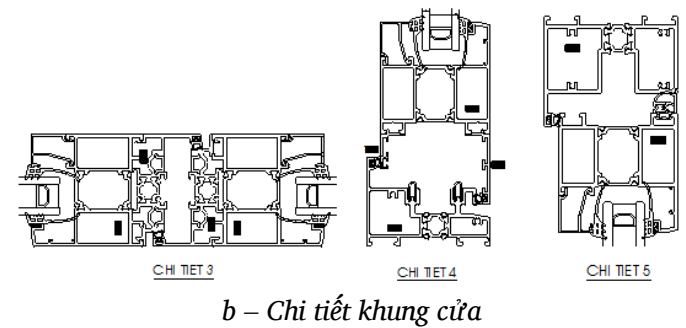

Hình 3. Chi tiết sản phẩm mẫu cửa sổ.

\subsection{Phần mềm tính toán}

\subsubsection{Phần mềm WINDOW}

Độ truyền nhiệt của sản phẩm cửa qua phần kính trung tâm chủ yếu là quá trình một chiều. Nó được phân tích bằng cách chia nhỏ mặt cắt ngang của hệ thống kính thành một tổ hợp các nút và tính toán sự truyền nhiệt giữa mỗi nút. Trong điều kiện trạng thái ổn định, dòng năng lượng đi vào mỗi nút bằng với dòng năng lượng đi ra khỏi mỗi nút. Để thực hiện cân bằng năng lượng, WINDOW mô hình hóa hệ thống kính do người dùng xác định như một mạng lưới tấm cách nhiệt một chiều trong trạng thái ổn định, được hiển thị trong Hình 4. Sử dụng phương pháp lặp để hội tụ phân bố nhiệt độ chính xác. Từ sự phân bố nhiệt độ này, có thể tính được bất kỳ chỉ số tính năng mong muốn nào [5].

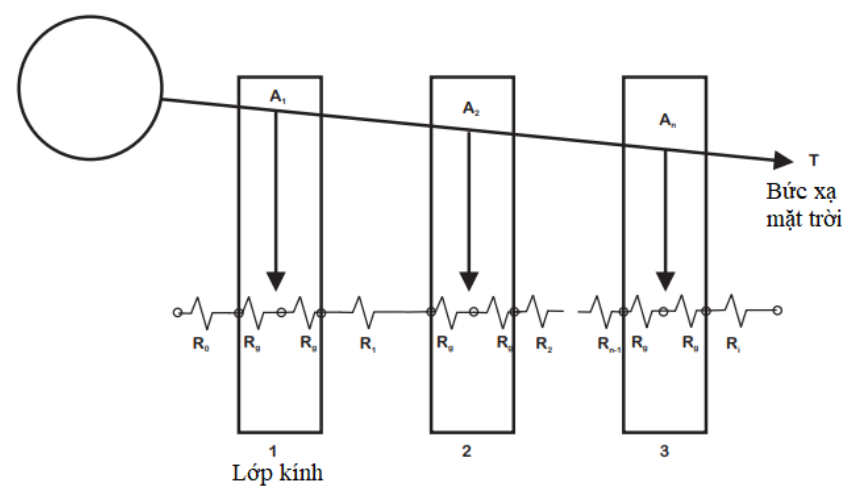

Hình 4. Mạng nút nhiệt trở sử dụng để mô hình phần tâm kính trong Window.

Hệ số truyền nhiệt dẫn điện/đối lưu được tính toán dựa trên các phương trình thực nghiệm. Lớp màng không khí tiếp xúc bên ngoài phụ thuộc vào tốc độ gió và hướng gió thổi. Lớp màng không khí tiếp xúc bên trong là một hàm của chênh lệch nhiệt độ bề mặt hướng vào trong, nhiệt độ trong nhà và chiều cao của sản phẩm cửa. Hệ số truyền nhiệt trong khoang khí được tính từ phương trình thực nghiệm Nusselt. Chuẩn số Nusselt là một đại lượng không thứ nguyên có liên quan đến sự chênh lệch nhiệt độ giữa các bề mặt giới hạn trong khoang khí, và chiều rộng, chiều cao và tính chất nhiệt của khoang khí. Độ nghiêng của sản phẩm cửa cũng được tính đến trong tất cả các mối tương quan đến dẫn nhiệt/đối lưu của sản phẩm [5].

Hệ số truyền nhiệt bức xạ hiệu quả là tỷ lệ giữa thông lượng bức xạ giữa các nút bức xạ và chênh lệch nhiệt độ liên quan. Độ truyền nhiệt qua sản phẩm của là hệ số U-value tổng của của, là đại lượng nghịch đảo của tổng nhiệt trở trong trường hợp không có bức xạ mặt trời. Phép đo độ truyền năng lượng do bức xạ mặt trời là Hệ số hấp thụ năng lượng mặt trời SHGC, được tính toán từ đặc tính quang học của hệ thống kính và khung, và phần năng lượng mặt trời hấp thụ được truyền tới phía trong nhà.

Để mô hình hóa chính xác các hệ thống kính nhiều lớp kính chọn lọc quang phổ (tức là kính có các đặc tính đơn hướng thay đổi theo bước sóng, chẳng hạn như kính nhiều lớp phủ có độ phát xạ thấp kính lowE), một mô hình đa tần được sử dụng trong WINDOW. Trong mô hình này, WINDOW tính toán độ truyền qua và độ phản xạ cho lớp kính theo bước sóng, và sau đó cân bằng các tính chất bởi các trọng số chức năng để có được tổng các đặc tính về bức xạ mặt trời, khả kiến, nhiệt hồng ngoại, cũng như các trọng số tổn thất hệ số truyền và hệ số truyền từ 0,30 đến 0,38 micrômét. Để sử dụng mô hình đa tần này, WINDOW cần một tệp dữ liệu quang phổ cho mỗi lớp kính. Các tệp dữ liệu này được cập nhật và duy trì bởi LBNL và có sẵn từ NFRC. Nếu lớp kính trong hệ thống kính không có tệp dữ liệu quang phổ, WINDOW sử dụng quang phổ phẳng của kính mà không có tệp dữ liệu quang phổ, dựa trên các tính chất khả kiến và bức xạ năng lượng mặt trời [5].

\subsubsection{Phần mềm THERM}

THERM kết hợp mô hình truyền nhiệt hai chiều, sử dụng phương pháp phần tử hữu hạn để số hóa phương trình năng lượng hai chiều điều chỉnh. Hình dạng của hệ thống cửa bao gồm khung và các phần kính, vật liệu kính được chuyển từ phần mềm WINDOW. Vật liệu khung được thực hiện trong chương trình THERM và các điều kiện biên thích hợp được áp dụng trên các bề mặt khung tiếp xúc với không khí trong nhà và không khí ngoài trời, trong khi các bề mặt khung dưới cùng và trên cùng của bề mặt kính được chỉ định là các điều kiện biên đoạn nhiệt. dưới cùng và trên cùng của bề mặt kính được chỉ định là các điều kiện biên đoạn nhiệt. Mô hình số cần phải các điều kiện biên được gán cho mỗi bề mặt biên của mô hình để được xác định hoàn toàn. Các điều kiện ranh giới trên bề mặt trong nhà và ngoài trời bao gồm cả thành phần đối lưu và bức xạ. Thành phần đối lưu ở phía trong nhà được xác định thông qua việc sử dụng nhiệt độ phụ thuộc vào hệ số truyền nhiệt bề mặt, dựa trên mối tương quan đối lưu tự nhiên. Đối với mỗi loại vật liệu khung có một giá trị hệ số truyền nhiệt bề mặt đối lưu không đổi. Thành phần nhiệt bức xạ được mô hình hóa thông qua việc sử dụng mô hình bức xạ chi tiết dựa trên hệ số quan sát. Độ dẫn nhiệt hiệu quả của kính được chuyển từ phần mềm WINDOW, trong khi đối với các khoang khí của khung của được chương trình THERM tính toán dựa trên hình dạng hình 
học, hướng dòng nhiệt, độ phát xạ và nhiệt độ bề mặt. Các khoang khí của khung cửa có thể được bao bọc hoàn toàn hoặc thông gió một phần (chỉ cho phần phía ngoài trời), tùy thuộc vào cấu hình và kích thước của các thành phần, kết nối khoang khí của khung với môi trường bên ngoài [5].

Sau khi hoàn thành mô phỏng số hóa, phần ước tính lỗi của chương trình sẽ tính toán lỗi và trên cơ sở mức độ lỗi cần tinh chỉnh các vùng lỗi của mô hình và tính toán lại toàn bộ mô hình. Quy trình này được lặp lại cho đến khi không có vùng cục bộ nào hiển thị mức lỗi cao hơn mức quy định. Công cụ ước tính lỗi này dựa trên phương pháp định mức sai số năng lượng của Zinkiewitz [5].

\subsubsection{Phần mềm OPTICS}

Optics là phần mềm được thiết kế để xử lý, tính toán các dữ liệu quang học của lớp kính và sản phẩm kính. Phần mềm sử dụng các dữ liệu truyền và phản xạ quang phổ bức xạ mặt trời theo bước sóng. Điều này cho phép việc tính toán các giá trị tổng thể của sản phẩm cửa như độ truyền sáng (VLT), độ hấp thụ năng lượng mặt trời (SHGC),... theo nhiều tiêu chuẩn khác nhau [6].

Dữ liệu tính toán sử dụng trong phần mềm là dữ liệu đo của các thành phần kính đơn, màng dán kính,... để tạo ra các sản phẩm kính nhiều lớp và tính toán các giá trị quang phổ cho hệ thống kết hợp từ dữ liệu quang phổ. Các đặc tính năng lượng mặt trời có thể tính toán được như: Hệ số truyền sáng $\left(\mathrm{T}_{\mathrm{vis}}\right)$; Hệ số truyền bức xạ mặt trời $\left(\mathrm{T}_{\mathrm{sol}}\right)$; Hệ số phản xạ ánh sáng nhìn thấy $\left(\mathrm{R}_{\mathrm{vis}}\right)$; Hệ số phản xạ bức xạ mặt trời $\left(\mathrm{R}_{\mathrm{sol}}\right)$; Hệ số phát xạ nhiệt $(\varepsilon)$; các hệ số liên quan tới bảo vệ chống UV như Hệ số truyền tia cực tím $\left(\mathrm{T}_{\mathrm{UV}}\right)$; Hệ số bảo vệ trước bức xạ mặt trời (SPF); Hệ số bức xạ nguy hại truyền qua $\left(\mathrm{T}_{\mathrm{DW}}\right), \ldots$ [6]

\subsection{Phương pháp nghiên cứu}

\subsubsection{Phương pháp tính toán và thử nghiệm}

- Kiểm tra tính phù hợp của phần mềm thông qua mô phỏng mẫu có kích thước, thông số được đưa ra trong phụ lục $\mathrm{D}$, TCVN 11857:2017 và phụ lục D, TCVN 10077-2:2012;

- Tính toán cho phần khung cửa: ISO 10077-2:2017 Đặc trưng nhiệt của cửa sổ, cửa ra vào và cửa chớp - Tính toán truyền nhiệt Phần 2: Phương pháp số cho khung cửa;

- Tính toán cho toàn bộ cửa: TCVN 11857:2017 (ISO 15099:2003) Đặc trưng nhiệt của cửa sổ, cửa đi và kết cấu che nắng Tính toán chi tiết;

- Tính chất truyền quang và truyền bức xạ mặt trời của tấm kính đơn, rèm được xác định bằng máy quang phổ JASCO V-770 UVVis/NIR Spectrophotometer theo TCVN 7737:2007 (ISO 9050:2003);

- Tính chất phát xạ nhiệt của kính đơn và vật liệu rèm được xác định bằng máy quang phổ JASCO FT/IR-6600 FT-IR Spectrometer theo ISO 10292:1994

\subsubsection{Phiên bản phần mềm mô phỏng sử dụng}

Sau khi đo đạc xác định các đặc tính truyền quang \& truyền bức xạ mặt trời của vật liệu theo phương pháp tính toán và thử nghiệm, các thông số này được đưa vào phần mềm mô phòng như Window V7.7.10; Therm V7.7.10 và Optic V6.0 được cung cấp bởi LBNL để xác định thông số đặc trưng nhiệt của toàn bộ hệ cửa sổ, cửa đi và kết cấu che nắng.

\section{Kết quả và thảo luận}

\subsection{Nghiên cứu tính phù hợp của phần mềm WINDOW}

Để xác nhận phần mềm WINDOW 7.7 tuần thủ theo TCVN 11857 (ISO 15099), tiến hành tính toán mô phỏng bằng phần mềm các ví dụ xác nhận được cung cấp trong Phụ lục $\mathrm{D}$ của tiêu chuẩn TCVN 11857 (ISO 15099), NFRC 100. Bảng 2 trình bày tóm tắt các kết quả tính toán hiệu suất nhiệt bằng phần mềm mô phỏng WINDOW 7.7 và so sánh với kết quả thể hiện trong Ví dụ được đưa ra trong tiêu chuẩn TCVN 11857 (ISO 15099).

Bảng 2. Tóm tắt kết quả mô phỏng so sánh với kết quả được tính toán theo tiêu chuẩn.

\begin{tabular}{|c|c|c|c|c|c|c|}
\hline \multirow[b]{2}{*}{ Ví dụ } & \multicolumn{3}{|c|}{$U$-value } & \multicolumn{3}{|l|}{ SHGC } \\
\hline & $\begin{array}{l}\text { TCVN } \\
11857\end{array}$ & WINDOW 7.7 & $\%$ & $\begin{array}{l}\text { TCVN } \\
11857\end{array}$ & WINDOW 7.7 & $\%$ \\
\hline D.3.1 & 5,690 & 5,689 & $-0,02$ & 0,574 & 0,570 & $-0,70$ \\
\hline D.3.2 & 2,850 & 2,827 & $-0,70$ & 0,791 & 0,779 & $-1,52$ \\
\hline
\end{tabular}

Bảng 3. Tóm tắt kết quả toàn bộ cửa so sánh với kết quả được tính toán theo tiêu chuẩn.

\begin{tabular}{|c|c|c|c|c|c|c|}
\hline \multirow{2}{*}{ Ví dụ } & \multicolumn{3}{|c|}{ U-value } & \multicolumn{3}{c|}{ SHGC } \\
\cline { 2 - 7 } & $\begin{array}{c}\text { TCVN } \\
11857\end{array}$ & WINDOW 7.7 & $\%$ & $\begin{array}{c}\text { TCVN } \\
11857\end{array}$ & WINDOW 7.7 & $\%$ \\
\hline D.4.1 & 5,20 & $\mathbf{5 , 2 2 7}$ & 0,52 & 0,480 & $\mathbf{0 , 4 7 9}$ & $-0,21$ \\
\hline D.4.2 & 3,00 & $\mathbf{2 , 9 5 2}$ & $-1,6$ & 0,660 & $\mathbf{0 , 6 5 1}$ & $-1,36$ \\
\hline
\end{tabular}

Kết quả cho thấy các thông số sử dụng phần mềm mô phỏng WINDOW 7.7 phù hợp với giá trị công bố trong TCVN 11857:2017.

\subsection{Nghiên cứu tính phù hợp của phần mềm THERM}

Để xác nhận phần mềm THERM 7.7 tuần thủ theo ISO 100772:2017, tiến hành tính toán mô phỏng bằng phần mềm các ví dụ xác nhận được cung cấp trong Phụ lục $\mathrm{D}$ của tiêu chuẩn. Bảng 1 trình bày tóm tắt các kết quả tính toán hiệu suất nhiệt. Theo Điều 4.2 của ISO 10077-2, phần mềm được xác thực phải có khả năng tính toán độ dẫn nhiệt hai chiều, $\mathrm{L}^{2 \mathrm{D}}$, của các ví dụ được cung cấp trong Phụ lục $\mathrm{D}$ trong phạm vi $\pm 3 \%$ của các giá trị được đưa ra trong bảng. $L^{2 D}$ được mô phỏng cho tất cả các ví dụ được mô hình hóa nằm trong khoảng $3 \%$ của giá trị được chấp nhận. Mặc dù không phải là 
một tiêu chí xác nhận, nhưng $U_{f}$ được mô phỏng cho tất cả các ví dụ được mô hình hóa trong khoảng $5 \%$ giá trị dự kiến.

Bảng 5. Tóm tắt kết kết quả mô phỏng so sánh với kết quả được tính toán theo tiêu chuẩn.

\begin{tabular}{|c|c|c|c|c|c|c|}
\hline \multirow{2}{*}{ Ví du } & \multicolumn{3}{|c|}{$L^{2 D}(W / m . K)$} & \multicolumn{3}{c|}{$U_{f}\left(W / m^{2} . K\right)$} \\
\cline { 2 - 7 } & ISO 10077 & THERM 7.7 & $\%$ & ISO 10077 & THERM 7.7 & $\%$ \\
\hline D.1 & 0,550 & $\mathbf{0 , 5 5 4}$ & 0,7 & 3,22 & $\mathbf{3 , 2 5}$ & 0,9 \\
\hline D.2 & 0,263 & $\mathbf{0 , 2 6 2}$ & $-0,4$ & 1,44 & $\mathbf{1 , 4 3}$ & $-0,7$ \\
\hline D.3 & 0,424 & $\mathbf{0 , 4 2 9}$ & 1,2 & 2,07 & $\mathbf{2 , 1 2}$ & 2,4 \\
\hline D.4 & 0,346 & $\mathbf{0 , 3 4 7}$ & 0,3 & 1,36 & $\mathbf{1 , 3 6}$ & 0 \\
\hline D.5 & 0,408 & $\mathbf{0 , 4 0 2}$ & $-1,5$ & 2,08 & $\mathbf{2 , 0 2}$ & $-2,9$ \\
\hline D.6 & 0,659 & $\mathbf{0 , 6 4 7}$ & $-1,8$ & 4,67 & $\mathbf{4 , 5 5}$ & $-2,6$ \\
\hline D.7 & 0,285 & $\mathbf{0 , 2 8 4}$ & $-0,4$ & 1,31 & $\mathbf{1 , 2 8}$ & $-2,3$ \\
\hline
\end{tabular}

- Tính toán $\mathrm{U}_{\mathrm{f}}$ : Theo các phương pháp sử dụng các điều kiện biên tham chiếu trong Bảng D1 và tính chất vật liệu trong Bảng D2 được nêu trong tiêu chuẩn ISO 10077-2, độ dẫn nhiệt của mô hình $\mathrm{L}_{\mathrm{f}}^{2 \mathrm{D}}$ được tính toán với giá trị nằm trong khoảng $\pm 3 \%$ giá trị được tính toán theo tiêu chuẩn:

\begin{tabular}{|l|l|l|l|}
\hline Thông số & ISO 10077-2:2017 & THERM 7.7 & \% sai lệch \\
\hline $\mathrm{L}_{\mathrm{f}}^{2 \mathrm{D}}(\mathrm{W} / \mathrm{mK})$ & $0,346(0,001)$ & $\mathbf{0 , 3 4 7}$ & $0,3 \%$ \\
\hline $\mathrm{U}_{\mathrm{f}}\left(\mathrm{W} / \mathrm{m}^{2} \mathrm{~K}\right)$ & $1,36(0,01)$ & $\mathbf{1 , 3 7}$ & $0,7 \%$ \\
\hline
\end{tabular}

Từ những so sánh trên cho thấy phần mềm Therm đưa ra kết quả đáp ứng mức sai lệch cho phép của tiêu chuẩn.

3.3. Nghiên cứu tính toán xác định các đặc trưng nhiệt của hệ thống cửa nhôm cầu nhiệt

\subsubsection{Tính toán đặc tính truyền nhiệt cho phần tâm kính}

Phần tâm kính là loại kính hộp hai lớp với mặt bên ngoài là lớp kính lowE, lớp kính bên trong là kính trắng và giữa hai lớp kính là khoang khí chứa 90 \% khí Argon. Phần dữ liệu tính toán của kính đơn được đo đạc thông qua máy quang phổ và sử dụng phần mềm Optics để xác định các đặc tính nhiệt và quang của từng lớp kính (Hình 5).

Điều kiện biên sử dụng khi tính toán đặc trưng nhiệt của kêt cấu cửa sử dụng điều kiện biên tham chiếu qui định trong Điều 8.2 của TCVN 11857.

Kết quả tính toán các thông số đặc trưng nhiệt của kính hộp được thể hiện trong Hình 7.
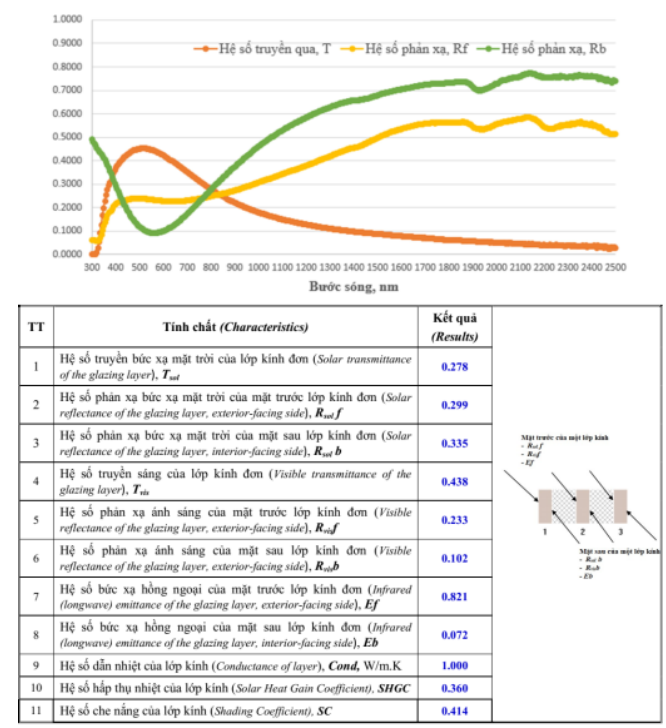

Hình 5. Đặc tính nhiệt, quang của lớp kính lowE.

\begin{tabular}{|c|c|}
\hline Điều kiện mùa đông (Winter conditions) & Điều kiện mùa hè (Summer conditions) \\
\hline 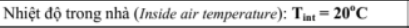 & 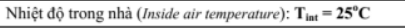 \\
\hline Nhiệt độ ngoài trời (Outside air temperature): $\mathrm{T}_{\mathrm{ex}}=0^{\circ} \mathrm{C}$ & Nhiệt độ ngoài trời (Outside air temperature): $\mathrm{T}_{\mathrm{ex}}=30^{\circ} \mathrm{C}$ \\
\hline $\begin{array}{l}\text { Hệ số trao đổi nhiệt đối lưu trong nhà (Inside convection } \\
\text { coefficient): } \mathbf{h}_{\mathrm{c} v \text {, iat }}=\mathbf{3 , 6} \mathbf{W} / \mathbf{m}^{2} . \mathbf{K}\end{array}$ & $\begin{array}{l}\text { Hệ số trao đồi nhiệt đối lưu trong nhà (Inside convection } \\
\text { coefficient): } \mathbf{h}_{\mathrm{er}, \text {,at }}=\mathbf{2 , 5} \mathbf{W} / \mathbf{m}^{2} . \mathbf{K}\end{array}$ \\
\hline $\begin{array}{l}\text { Hệ số trao đổi nhiệ̂ đối lưu ngoài nhà (Ousside convection } \\
\text { coefficient): } \mathbf{h}_{\mathrm{c}, \mathrm{er}}=\mathbf{2 0} \mathbf{W} / \mathrm{m}^{2} . \mathbf{K}\end{array}$ & $\begin{array}{l}\text { Hệ số trao đổi nhiệt đối lưu ngoài nhà (Ousside convection } \\
\text { coefficient): } \mathbf{h}_{\mathrm{s} v, \mathrm{ea}}=\mathbf{8} \mathbf{W} / \mathbf{m}^{2} \cdot \mathbf{K}\end{array}$ \\
\hline Tốc độ gió ngoài trời (Outside wind speed): $\mathbf{v}=\mathbf{4}$ m/s & Tốc độ gió ngoài trời (Outside wind speed): $\mathbf{v}=1 \mathrm{~m} / \mathbf{s}$ \\
\hline $\begin{array}{l}\text { Nhiệt độ bức xạ bên ngoài nhà (Effective sky temperature): } \\
\mathbf{T}_{r, \mathrm{~m}}=\mathbf{T}_{e x}\end{array}$ & $\begin{array}{l}\text { Nhiệt độ bức xạ bên ngoài nhà (Effective sky temperature): } \\
\mathbf{T}_{\mathrm{r}, \mathrm{m}}=\mathbf{T}_{\mathrm{ex}}\end{array}$ \\
\hline $\begin{array}{l}\text { Nhiệt độ bức xạ bên trong nhà (Effective room temperature): } \\
\mathbf{T}_{r, \mathrm{~m}}=\mathbf{T}_{\text {int }}\end{array}$ & $\begin{array}{l}\text { Nhiệt độ bức xạ bên trong nhà (Effective room temperature): } \\
\mathbf{T}_{r, \mathrm{~m}}=\mathbf{T}_{\text {iat }}\end{array}$ \\
\hline $\begin{array}{l}\text { Cường độ bức xạ mặt trời (Direct solar radiation): } \\
\mathbf{I}_{\mathbf{s}}=\mathbf{3 0 0} \mathbf{W} / \mathbf{m}^{2}\end{array}$ & $\begin{array}{l}\text { Cường độ bức xạ mặt trời (Direct solar radiation): } \\
\mathbf{I}_{\mathbf{s}}=\mathbf{5 0 0} \mathbf{W} / \mathrm{m}^{2}\end{array}$ \\
\hline
\end{tabular}

Hình 6. Điều kiện biên sử dụng trong tính toán.

\begin{tabular}{|c|c|c|c|c|c|c|c|c|c|}
\hline \multirow{2}{*}{\multicolumn{2}{|c|}{$\begin{array}{l}\text { Ufrecta } \\
\text { W/m2K }\end{array}$}} & sc & SHGC & Rel He Gan & Tvis & Keff & Laser 1 Keff & Gap 1 Keff & Layer 2 Keff \\
\hline & & & & W/m2 & & W/mk & W/mk & W/mKK & W/mk \\
\hline \multicolumn{2}{|c|}{1.405} & 0.353 & 0307 & 234 & 0.395 & 0.0438 & 1.0000 & 0.0224 & 1.0000 \\
\hline TT & \multicolumn{7}{|c|}{ Tính chất (Characteristics) } & $\begin{array}{l}\text { Kết quãa } \\
\text { (Results) }\end{array}$ & $\begin{array}{l}\text { Đon vi } \\
\text { (Units) }\end{array}$ \\
\hline 1 & \multicolumn{7}{|c|}{ Hệ số truyền nhiệt (Thermal transmittance), $\boldsymbol{U}$-value } & 1.405 & $\mathrm{~W} / \mathrm{m}^{2} \mathrm{~K}$ \\
\hline 2 & \multicolumn{7}{|c|}{ Hệ số che nắng (Shading Coefficient), SC } & 0.353 & - \\
\hline 3 & \multicolumn{7}{|c|}{ Hệ số hấp thụ nhiệt mặt trời (Solar Heat Gain Coefficient), SHGC } & 0.307 & - \\
\hline 4 & \multicolumn{7}{|c|}{ Nhiệt lượng hấp thụ tương đối (Relative Heat Gain), Rel. Ht. Gain } & 234 & $\mathrm{~W} / \mathrm{m}^{2}$ \\
\hline 5 & \multicolumn{7}{|c|}{ Hệ số truyển sáng (Visible transmittance), Tvis (VLT) } & 0.395 & $\cdot$ \\
\hline 6 & \multicolumn{7}{|c|}{ Hệ số dẫn nhiệt hiệu quả (Effective conductivity), Keff } & 0.0438 & $\mathrm{~W} / \mathrm{mK}$ \\
\hline
\end{tabular}

Hình 7. Kết quả tính toán đặc trưng nhiệt của kính hộp.

\subsubsection{Tính toán đặc tính truyền nhiệt cho phần khung cửa}

Phần khung bao cửa là dạng thanh nhôm định hình có kết cấu profile khác nhau ở những vị trí khác nhau trên cửa. Để tính toán được thông số đặc trưng nhiệt của toàn bộ kết cấu cửa thì cần thực hiện mô phỏng toàn bộ các chi tiết profile có trong kết cấu cửa sử dụng khi tính toán cụ thể như sau:

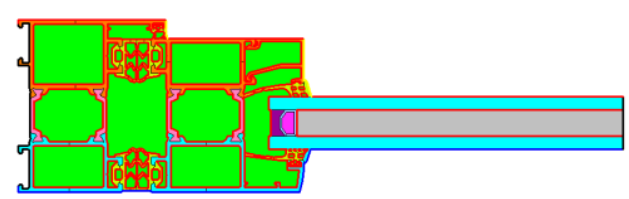


Thông tin vật liệu sử dụng để đưa vào phần mềm tính toán như sau:

\begin{tabular}{|c|l|c|c|c|c|}
\hline STT & \multicolumn{1}{|c|}{ Tên cấu kiện } & Vật liệu & $\lambda$ (W/m.K) & $\varepsilon$ & Ghi chú \\
\hline 1 & $\begin{array}{l}\text { Khung bao cửa } \\
\text { đi/7003i }\end{array}$ & Al6063-T5 & 209 & 0,918 & \\
\hline 2 & $\begin{array}{l}\text { Cánh cửa } \\
\text { đi/7004i }\end{array}$ & Al6063-T5 & 209 & 0,921 & \\
\hline 3 & Gioăng & EPDM & 0,25 & 0,900 & \\
\hline 4 & Cầu cách nhiệt & Polyamide & 0,30 & 0,900 & \\
\hline 5 & Chất hút ầm & Silica gel & 0,13 & 0,900 & \\
\hline 6 & Cữ kính & $\begin{array}{c}\text { Steel-stainless } \\
\text { (buffed) }\end{array}$ & 17,0 & 0,200 & \\
\hline 7 & Keo bịt kín & Silicone & 0,35 & 0,9 & \\
\hline 8 & $\begin{array}{l}\text { Kính hộp lowE - } \\
\text { dày 24mm }\end{array}$ & - & - & - & \\
\hline
\end{tabular}

Điều kiện biên của mẫu:

\begin{tabular}{|c|l|c|c|c|c|}
\hline$S T T$ & Mô tả & $q\left(W / m^{2}\right)$ & $\theta\left({ }^{\circ} \mathbf{C}\right)$ & $R\left(m^{2} \cdot K / W\right)$ & Ghi chú \\
\hline 1 & Bên ngoài & & 0 & 0,040 & \\
\hline 2 & $\begin{array}{l}\text { Bên trong } \\
\text { giảm }\end{array}$ & & 20 & 0,20 & \\
\hline 3 & $\begin{array}{l}\text { Bên trong } \\
\text { chuẩn }\end{array}$ & & 20 & 0,13 & \\
\hline 4 & Đoạn nhiệt & 0,0 & & & \\
\hline
\end{tabular}

Kết quả tính toánmô phỏng đặc tính nhiệt bởi phần mềm Therm đưa ra như sau:

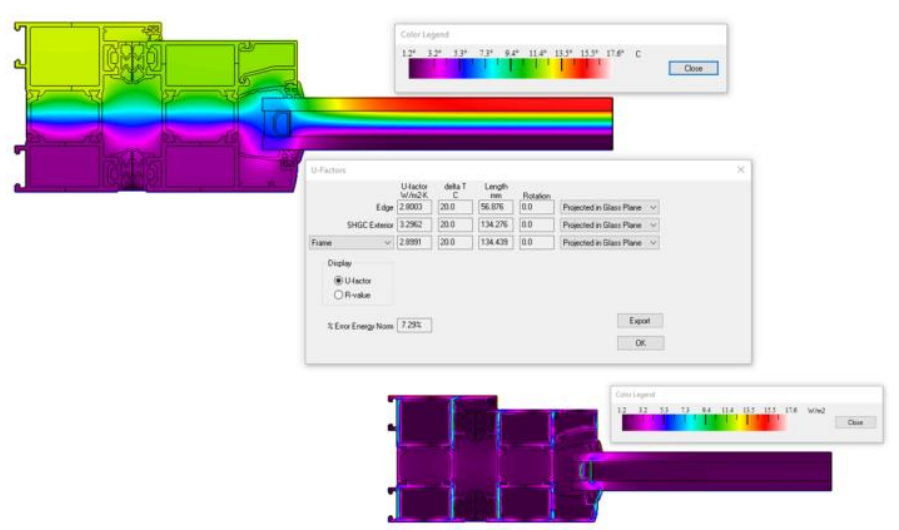

Hình 8. Kết quả đặc trưng nhiệt phần khung.

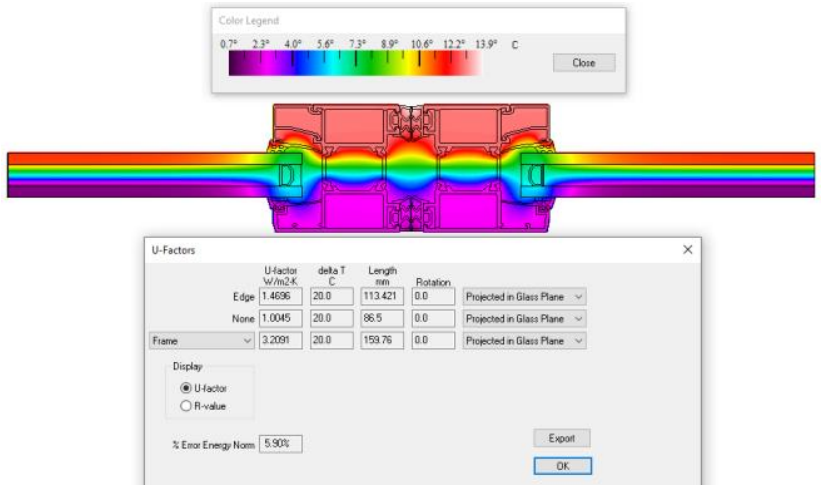

Hình 9. Kết quả đặc trưng nhiệt của khung chia.

\subsubsection{Tính toán đặc tính truyền nhiệt cho toàn bộ hệ thống cửa}

Sau khi mô phỏng tất cả các chi tiết của khung cửa, tiến hành đưa các chi tiết này vào phần mềm WINDOW xác định đặc trưng nhiệt của toàn bộ kết cấu cửa sổ như Hình 10 .

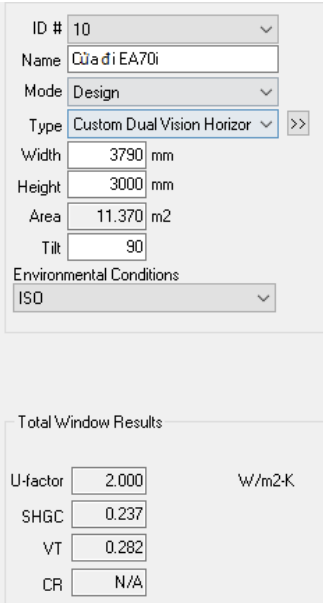

Hình 10. Kết quả tính các thông số đặc trưng nhiệt của toàn bộ cửa sổ cầu trượt dùng kính LowE trên phần mềm WINDOW.

Thông số đặc trưng nhiệt của cửa thu được là các giá trị Uvalue $=2,00 \mathrm{~W} / \mathrm{m}^{2} . \mathrm{K} ; \mathrm{SHGC}=0,237$ và $\mathrm{VLT}=0,282$. Các đặc trưng nhiệt này được sử dụng cho mục đích thiết kế công trình tuân thủ Quy chuẩn kỹ thuật quốc gia về các công trình sử dụng năng lượng hiệu quả QCVN 09:2017/BXD.

\begin{tabular}{|c|c|c|c|}
\hline \multirow{2}{*}{ WWR (\%) } & \multicolumn{3}{|c|}{ SHGC $_{\max }$} \\
\hline $\mathbf{2 0}$ & Hướng Bắc & Hướng Nam & Các hướng còn lại \\
\hline $\mathbf{3 0}$ & 0.90 & 0.90 & 0.80 \\
\hline $\mathbf{4 0}$ & 0.64 & 0.70 & 0.58 \\
\hline $\mathbf{5 0}$ & 0.50 & 0.56 & 0.46 \\
\hline $\mathbf{6 0}$ & 0.40 & 0.45 & 0.38 \\
\hline $\mathbf{7 0}$ & 0.33 & 0.39 & 0.32 \\
\hline $\mathbf{8 0}$ & 0.27 & 0.33 & 0.27 \\
\hline $\mathbf{9 0}$ & 0.23 & 0.28 & 0.23 \\
\hline $\mathbf{1 0 0}$ & 0.20 & 0.25 & 0.20 \\
\hline & 0.17 & 0.22 & 0.17 \\
\hline
\end{tabular}


3.4. Đánh giá hiệu quả tiết kiệm năng lượng so với cửa nhôm kính thông thường

Cửa sổ tiết kiệm năng lượng được biết đến là có thể giảm đáng kể chi phí sưởi ấm và làm mát tòa nhà đồng thời làm tăng tính tiện nghi cho người ở và giảm thiểu các vấn đề ngưng tụ hơi nước trên bề mặt cửa sổ. Tuy nhiên, người tiêu dùng thường bối rối về cách chọn cửa sổ hiệu quả nhất. Thông tin sản phẩm thường cung cấp các đặc trưng nhiệt của cửa sổ: Hệ số truyền nhiệt U-value hoặc nhiệt trở Rvalue, Hệ số hấp thụ nhiệt mặt trời SHGC hoặc Hệ số che nắng SC, và tỷ lệ rò rỉ không khí. Tuy nhiên, vai trò tiết kiệm năng lượng của những thông số đặc trưng này lại phụ thuộc vào điều kiện môi trường cụ thể và kết cấu tòa nhà. Hơn nữa, các tính chất này được tính toán và thử nghiệm theo các điều kiện đánh giá tĩnh được tiêu chuẩn hóa rất khác với điều kiện thực mà sản phẩm cửa sổ được ứng dụng tại công trình thực tế. Nhằm giúp người tiêu dùng và nhà xây dựng lựa chọn loại cửa có hiệu quả năng lượng tốt nhất về chi phí, nghiên cứu này đã thực hiện tính toán mô phỏng chi phí sử dụng năng lượng liên quan tới quá trình sưởi ấm và làm mát giữa hai loại sản phẩm cửa sổ cụ thể để làm căn cứ so sánh và đánh giá tham khảo.

Để thực hiện được việc này cần sử dụng công cụ mô phỏng máy tính nhằm tính toán lượng sử dụng năng lượng dùng cho sưởi ấm vào mùa đông và làm mát vào mùa hè, tính toán các chi phí điện liên quan cũng như nhu cầu sưởi ấm và làm mát cao điểm cho sản phẩm cửa cụ thể. Người dùng sẽ phải xác định một kịch bản cụ thể bằng cách lựa chọn một loại nhà (một tầng hoặc hai tầng) để mô phỏng, vị trí địa lý, hướng, đơn giá điện và chi tiết kết cấu nhà (chẳng hạn như tường, sàn và hệ thống điều hòa không khí HVAC).

Lựa chọn thiết lập Tòa nhà mô phỏng theo các thông số với những điều chỉnh tính toán cho điều kiện phù hợp với khí hậu Việt Nam như sau:

\section{- Vị trí: Hà Nội}

- Loại tòa nhà mô phỏng: Tòa nhà xây mới 1 tầng.

- Loại móng: Móng bê tông (Slab-On_Grade).

- Hệ thống làm mát HVAC: Bơm nhiệt chạy điện.

- Tổng diện tích sàn: 186 m².

- Tổng diện tích cửa sổ: $45 \mathrm{~m}^{2}$.

- Kết cấu bao che không sử dụng vật liệu cách nhiệt.

- Hệ số suy giảm bức xạ năng lượng mặt trời: Lựa chọn Typical.

- Nhiệt trở trần: R11.

- Nhiệt trở tường: R7.

- Đơn giá điện 2.535 đồng/kWh (0,110 \$/kWh).

3.4.1. Mô phỏng mức tiêu hao điện cho sưởi ấm và làm mát trong tòa nhà sử dụng cửa sổ nhôm kính thông thường

Cửa sổ nhôm kính thông thường với thông số đặc trưng nhiệt là: U-value là $5,618 \mathrm{~W} / \mathrm{m}^{2} \mathrm{~K}$; SHGC là 0,813 ; Độ rò rỉ không khí là
$1,52 \mathrm{l} / \mathrm{s} \cdot \mathrm{m}^{2}$. Kết quả chạy mô phỏng chi phí năng lượng tòa nhà mô phỏng trong 1 năm với khí hậu tại Hà Nội.

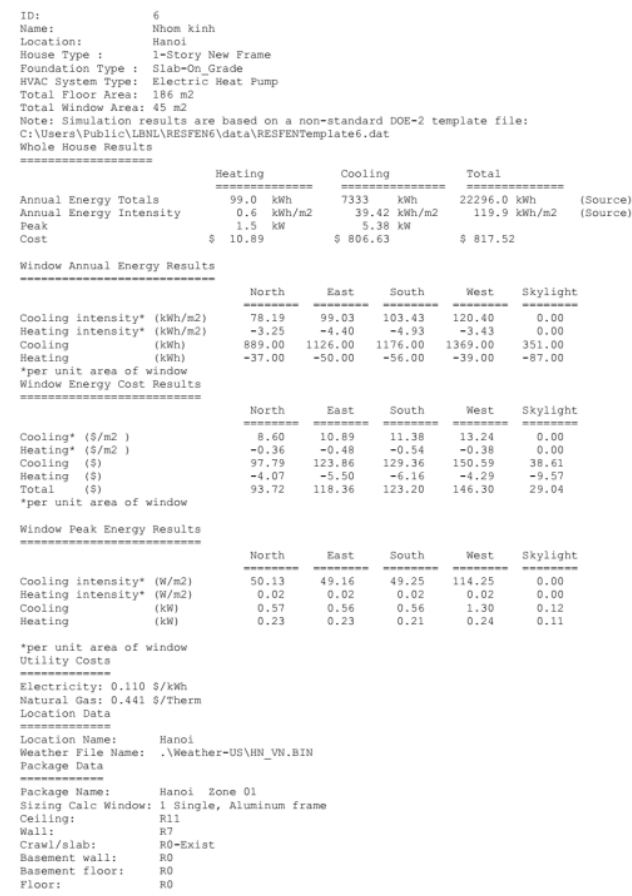

Hình 11. Chi phí năng lượng tòa nhà với cửa nhôm kính trong điều kiện khí hậu Hà Nội.

Cửa sổ nhôm cầu nhiệt kính hộp lowE với thông số đặc trưng nhiệt là: U-value là $2,000 \mathrm{~W} / \mathrm{m}^{2} \mathrm{~K}$; SHGC là 0,237 ; Độ rò rỉ không khí là $1,52 \mathrm{l} / \mathrm{s} \cdot \mathrm{m}^{2}$. Kết quả chạy mô phỏng chi phí năng lượng tòa nhà mô phỏng trong 1 năm với khí hậu tại Hà Nội.

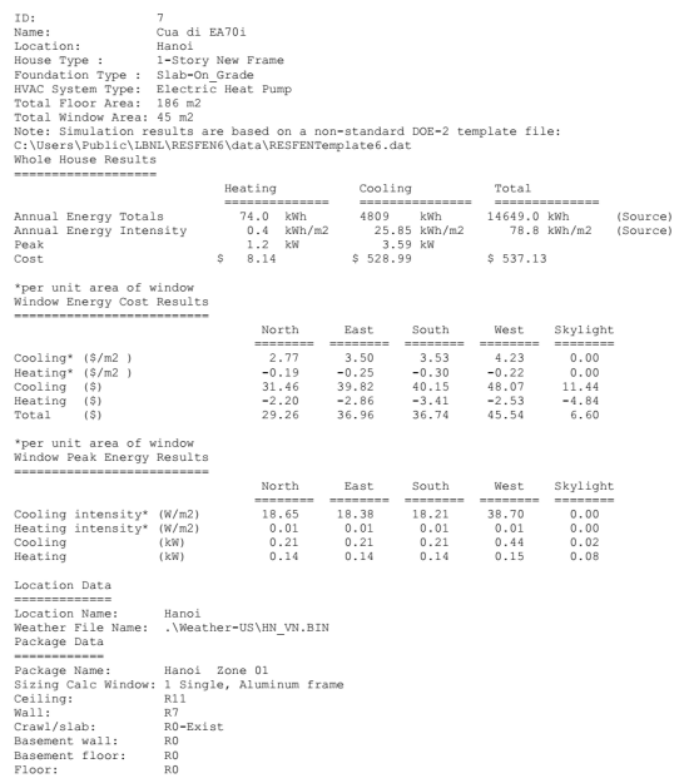

Hình 12. Chi phí năng lượng tòa nhà cửa tiết kiệm năng lượng trong điều kiện khí hậu Hà Nội. 
Kết quả so sánh chi phí sử dụng năng lượng điện cho điều hòa không khí tòa nhà mô phỏng sử dụng cửa nhôm kính cầu nhiệt có thể tiết kiệm được 34,3 \% chi phí điện/năm so với sử dụng cửa nhôm kính thông thường.

\begin{tabular}{|c|c|c|c|c|}
\hline TT & Loại cửa & $\begin{array}{c}\text { Chi phí } \\
\text { sưởi ấm } \\
\text { triệu } \\
\text { vnđ/năm }\end{array}$ & $\begin{array}{c}\text { Chi phí làm mát } \\
\text { triệu vnđ/năm }\end{array}$ & $\begin{array}{c}\text { Tổng chi phí } \\
\text { triệu vnđ/năm }\end{array}$ \\
\hline 1 & $\begin{array}{l}\text { Cửa nhôm } \\
\text { kính thông } \\
\text { thường }\end{array}$ & 0,249 & 18,448 & 18,697 \\
\hline 2 & $\begin{array}{l}\text { Cửa nhôm } \\
\text { cầu nhiệt } \\
\text { kính lowE }\end{array}$ & 0,186 & 12,098 & 12,284 \\
\hline $\begin{array}{l}\text { Chi phi tiết } \\
\text { kiệm }\end{array}$ & $\mathbf{0 , 0 6 3}$ & $\mathbf{6 , 3 5 0}$ & $\mathbf{6 , 4 1 3}$ \\
điện/năm
\end{tabular}

\section{Kết luận}

Nghiên cứu đã tiến hành đánh giá phần mềm THERM 7.7 và WINDOWN 7.7 có thuật toán đáp ứng các tiêu chuẩn tính toán TCVN 11857:2017; ISO 15099:2003; ANSI/NFRC 200-2020. Các kết quả tính toán trên cơ sở so sánh giá trị tính toán bằng phần mềm với giá trị công bố trong tiêu chuẩn đảm bảo sai lệch không quá $3 \%$ tuân thủ yêu cầu của tiêu chuẩn. Việc áp dụng phần mềm tính toán giúp đơn giản hóa các thuật toán phức tạp, dễ triển khai sử dụng và giảm thiểu các sai số do nhầm lẫn khi tính toán bằng thủ công.

Nghiên cứu cũng đã tiến hành tính toán xác định các thông số đặc trưng nhiệt cho sản phẩm cửa khung nhôm cầu nhiệt sử dụng kính hộp lowE. Kết quả cho thấy các thông số đặc trưng nhiệt được tính toán chi tiết theo các yêu cầu tiêu chuẩn. Nghiên cứu cũng đã tính toán mô phỏng chi phí sử dụng điện cho điều hòa không khí tòa nhà mô phỏng sử dụng cửa nhôm kính cầu nhiệt có thể tiết kiệm được 34,3 \% chi phí điện/năm so với sử dụng cửa nhôm kính thông thường

Trong tương lai nhóm nghiêm cứu đang hướng tới việc xây dựng tiêu chuẩn quốc gia TCVN và các chương trình dán nhãn cho những sản phẩm, vật liệu tiết kiệm năng lượng theo từng cấp bậc để người tiêu dùng dễ dàng nhận biết hiệu quả tiết kiệm năng lượng trong công trình. Bên cạnh đó nhóm nghiên cứu cũng sẽ mở rộng các hướng nghiên cứu tính toán các đặc trưng nhiệt cho các sản phẩm kính dán film cách nhiệt, rèm cửa và các kết cấu che nắng. Đồng thời nhóm cũng tiến hành nghiên cứu phát triển các sản phẩm, công nghệ mới có hiệu quả tiết kiệm năng lượng trong công trình xây dựng phù hợp với điều kiện khí hậu và điều kiện kinh tế kỹ thuật của Việt Nam.

\section{Tài liệu tham khảo}

[1]. Seok-Hyun Kim, Hakgeun Jeong, Soo Cho. "A study on changes of window thermal performance by analysis of physical test result in Korea", MDPI, 2019

[2]. Sang-Tae No, Jun-Sik Seo. "Analysis of Window components affecting $U$ value using thermal transmittance test results and multiple linear regression analysis", Hindawi, Advances in Civil Engineering, Volume 2018

[3]. Siu Fung Fung, Lin Lu. "Thermal performance analysis on different types of glazing of public rental housing in Hong Kong", $10^{\text {th }}$ International Symposion on Heating, Ventilation and Air conditionning, Elsevier Ltd, 2017

[4]. Carli, Inc. "Technical Report: Calculation of optical properties for a venetian blind type of shading device", 2006

[5]. National Fenestraion Rating Council, Inc. "Therm 7/Window 7 NFRC Simulation Manual", 2017

[6]. Richard Versluis, Rebecca Powles, Michael Rubin, "Optics version 5.1.01", LBNL Windows and Daylighting Group, 2002, https://windows.lbl.gov/software/

[7]. YourHome - Australia's independent guide to creating sustainable homes for the future, Glazing, from https://www.yourhome.gov.au/passivedesign/glazing, [truy cập ngày 26/10/2021]

[8]. ISO 10077-2:2003 Thermal Performance of windows, doors and shutters Calculation of thermal transmittance - Part 2: Numerical method for frames (Đặc trưng nhiệt của cửa sổ, cửa đi và cửa chớp - Tính toán truyền nhiệt - Phần 2: Phương pháp số cho khung cửa)

[9]. TCVN 11857:2017 (ISO 15099:2003), Đặc trưng nhiệt của cửa sổ, cửa đi và kết cấu che nắng - Tính toán chi tiết

[10]. TCVN 7737:2007 Kính xây dựng - Phương pháp xác định độ xuyên quang, độ phản quang, tổng năng lượng bức xạ mặt trời truyền qua và độ xuyên bức xạ tử ngoại

[11]. Tạp chí Kiến trúc số $07 / 2017$, Định mức năng lượng công trình - Bước khởi đầu hướng đến Kiến trúc tiết kiệm năng lượng, từ https://www.tapchikientruc.com.vn/chuyen-muc/dinh-muc-nang-luongcong-trinh-buoc-khoi-dau-huong-den-kien-truc-tiet-kiem-nang-luong.html, [truy cập ngày 26/10/2021]

[12]. Báo Công thương, Tiết kiệm năng lượng nhìn từ tòa nhà xanh, từ https://congthuong.vn/tiet-kiem-nang-luong-nhin-tu-toa-nha-xanh164273.html, [truy cập ngày 26/10/2021] 